\title{
Lidil
}

Revue de linguistique et de didactique des langues

\section{Gestion électronique des textes de lois consolidés :}

Une application du système générique de réédition personnalisée DARES

François Rousselot, Fady Farah et Amalia Todirascu

\section{(2) OpenEdition}

Journals

Édition électronique

URL : http://journals.openedition.org/lidil/2780

DOI : $10.4000 /$ lidil. 2780

ISSN : 1960-6052

Éditeur

UGA Éditions/Université Grenoble Alpes

Édition imprimée

Date de publication : 1 décembre 2008

Pagination : 89-108

ISBN : 978-2-84310-130-4

ISSN : $1146-6480$

Référence électronique

François Rousselot, Fady Farah et Amalia Todirascu, « Gestion électronique des textes de lois consolidés : », Lidil [En ligne], 38 | 2008, mis en ligne le 01 juin 2010, consulté le 19 avril 2019. URL: http://journals.openedition.org/lidil/2780 ; DOI : 10.4000/lidil.2780

Ce document a été généré automatiquement le 19 avril 2019

(c) Lidil 


\section{Gestion électronique des textes de lois consolidés :}

Une application du système générique de réédition personnalisée DARES

François Rousselot, Fady Farah et Amalia Todirascu

Avec l'accroissement du nombre de textes numérisés, qu'ilssoient disponibles ou non sur Internet, l'utilisateur qui effectue une recherche se trouve souvent devant une situation où les réponses qu'il obtient sont trop nombreuses pour être consultées exhaustivement. Il est alors intéressant de lui présenter un dossier fabriqué à partir des documents fournis en réponse à la requête, sous une forme qui condense les informations intéressantes qui s'y trouvent. Dans cette perspective, nous avons participé à un projet du Réseau National des Technologies Logicielles appelé PAPLOOํ. L'objectif était de concevoir et de mettre en application un système générique pour stocker et classer des documents, y accéder et enfin, permettre de les transformer pour produire une synthèse adaptée aux besoins des utilisateurs (Belaïd, 2005). La société participant au projet assure la maintenance de la base de données et l'accès à Eur-Lex (Logghe et al., 2000), site qui donne accès à une base de données des textes de lois communautaires. Elle nous a proposé, après avoir clairement identifié ce besoin chez les utilisateurs, de prendre comme application type la consultation des textes de lois, et spécialement les textes consolidés. L'objectif principal du projet était le développement d'un système générique fondé sur des outils réutilisables dans des domaines similaires. Nous aborderons peu ici l'aspect générique du système qui concerne plutôt la gestion de documents électroniques en général, nous nous focaliserons sur le domaine d'application.

Nous allons donc d'abord présenter les grandes lignes du projet PAPLOO, puis nous passerons à une description détaillée de l'application de PAPLOO aux textes de lois et conclurons sur les perspectives. 


\section{La chaine PAPLOO : vue d'ensemble}

L'hypothèse de base concernant le contexte d'application du système PAPLOO est qu'il s'adresse à une communauté d'utilisateurs qui ont les mêmes usages métier et qui par là même exécutent le même type de tâches de recherches sur des documents. Ceux-ci ont également des besoins similaires en ce qui concerne la présentation de la synthèse des résultats obtenus. Cette hypothèse permet d'envisager de fournir un ensemble de services prédéfinis pour résoudre leurs problèmes courants. Un utilisateur peut, en outre, préciser et personnaliser la façon d'effectuer la synthèse.

PAPLOO se présente sous la forme d'une chaine de traitements : dans une première phase les documents originaux bruts sont préparés, d'abord scannés puis passés à l'OCR ${ }^{2}$, s'il y a lieu, pour être finalement tous mis sous une forme normalisée en $\mathrm{XML}^{3}$. Les annotations XML qu'ils comportent se situent au niveau du document (méta-données). Une autre annotation concernera le découpage des textes en fragments et se situera donc à l'intérieur de chaque document.

Notre part dans la chaine de traitements de PAPLOO consiste à étiqueter sémantiquement (et logiquement) chaque document brut, pour fournir des documents structurés et annotés sémantiquement, puis à interpréter les annotations des documents répondant à la requête utilisateur pour lui fournir un dossier résultat (sous la forme de documents HTML $^{4}$ ici) qui lui permette de mieux les visualiser. Nous n'aborderons pas ici l'aspect gestion de profil utilisateur ni la tâche à effectuer pour adapter le système à une communauté particulière d'utilisateurs.

4 La chaine PAPLOO concerne la création d'une base de documents textuels de tout type qui sont préparés par une annotation externe (méta-données), puis interne (étiquetage des fragments à l'intérieur du document). Le système utilise un moteur de recherche qui sélectionne les documents répondant à une requête utilisateur. La présentation du dossier final est un ensemble de fichiers avec des liens hypertextes construits à partir des fragments étiquetés après interprétation de la sémantique des étiquettes.

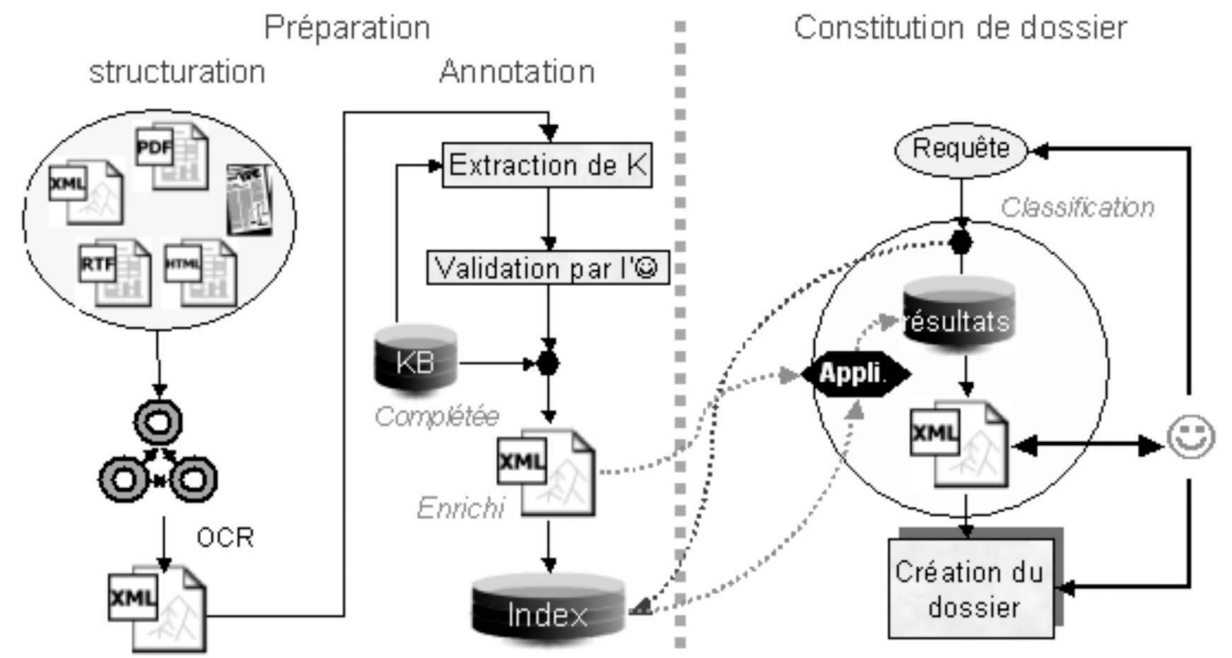


Figure 1 : Vue d'ensemble du système PAPLOO

5

(1)

« extraction de $\mathrm{K}$ » sur la figure 1 constituent le module appelé DARES ${ }^{6}$ que nous avons réalisé, notre contribution dans la chaine de traitement.

6 L'étiquetage sémantique a posé un problème particulier, en effet son rôle est non seulement de renseigner sur le contenu, mais également de signaler des liens sémantiques utilisables pour permettre ou pour suggérer des mises en page particulières. C'est ainsi que nous avons prévu des liens de généralisations/spécialisations qui peuvent être éventuellement utilisés pour présenter des fragments de documents avec différents niveaux d'indentation, pour suggérer un ordre hiérarchique ou, par exemple, des étiquettes à valeur temporelle qui pourront être utilisées pour présenter des résultats chronologiquement. De manière générale, un certain nombre d'étiquettes sont reliées à un ordre et peuvent être donc ordonnées. Elles sont utiles pour proposer des présentations différentes des fragments selon divers ordres. Dans l'application présentée ici, l'accent sera plutôt mis sur les annotions concernant des références à d'autres textes ou à d'autres parties de textes.

\section{DARES l'architecture}

DARES comporte deux parties indépendantes, le sous-module d'annotation qui effectue la phase de préparation, le sous-module de recombinaison qui construit, à partir des fragments obtenus, le dossier final.

\subsection{L'annotation}

Le module d'annotation utilise des règles d'annotation qui sont des combinaisons d'automates qui ont éventuellement des sorties (transducteurs). L'annotation se fait à deux niveaux: au niveau logique relativement à la structure définie par une valeur (donnée ou estimée) et au niveau sémantique qui dépend d'une phase d'étiquetage supplémentaire. Les étiquettes utilisées ici portent sur des informations pouvant servir de critères de sélection ou d'ordonnancement pour les fragments qui vont entrer dans la composition du document futur: liens de spécialisation/généralisation, annotations temporelles, liens propres au domaine comme, par exemple, dans le domaine juridique, l'expression qui introduit l'amendement ou certaines relations entre fragments (remplace, modifie, etc.).

7 Cet outil est fondé sur un langage défini pour exprimer des combinaisons non triviales d'automates (règles d'annotation). L'ordre d'utilisation des automates dans ces contextes et les sorties peuvent être indiqués pour les zones identifiées.

8 Nous présupposons l'existence dans le domaine d'une DTD $^{7}$ ou au moins d'éléments permettant d'accéder avec une bonne précision à la structure logique du texte.

9 L'outil d'annotation sert à convertir des documents TXT (en texte brut) ou XML en documents XML étiquetés logiquement et enrichis par l'information sémantique appropriée. Le jeu de règles d'annotation dépend évidemment de la langue. 


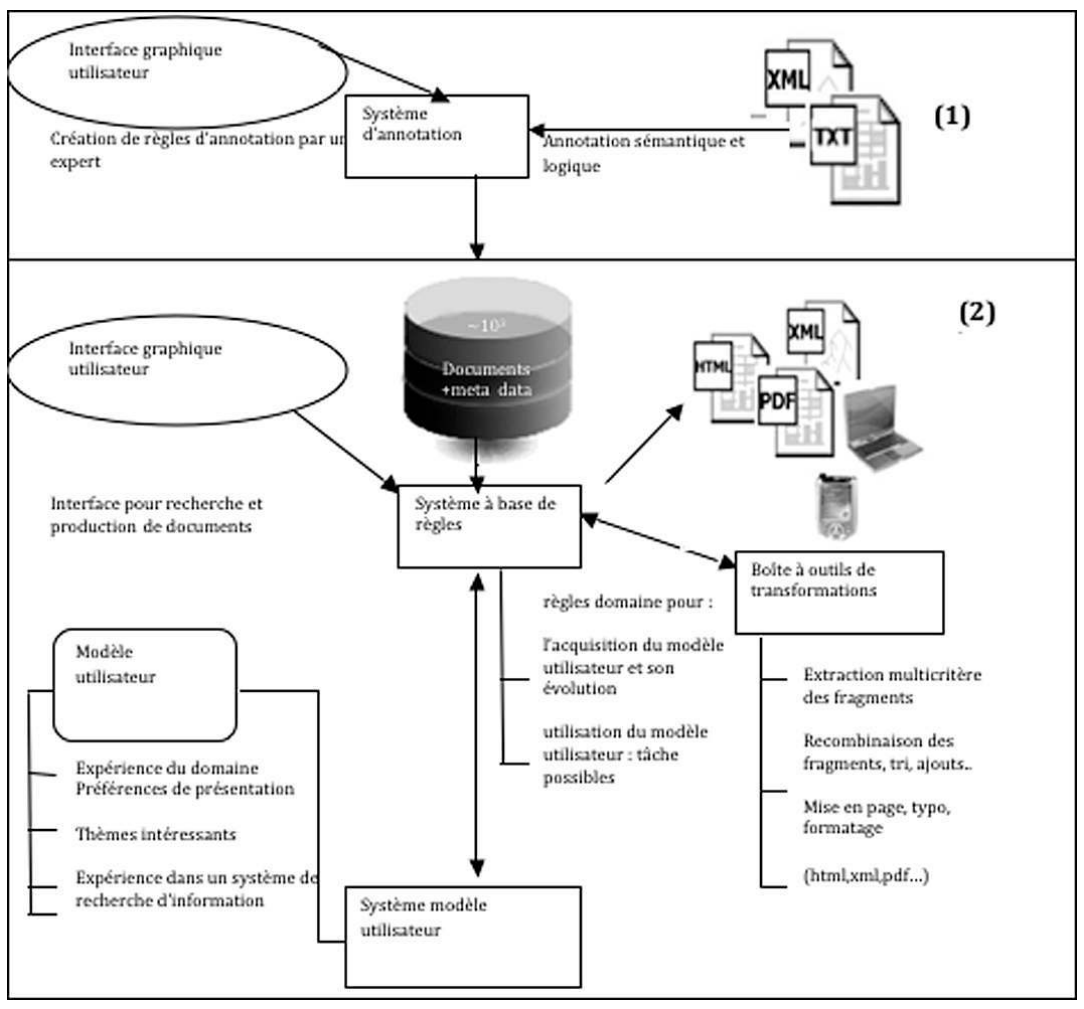

Figure 2 : Vue d'ensemble de l'annotation (1) et de la recombinaison (2)

\subsection{La recombinaison}

Ce module est constitué de trois parties :

- La boite à outils de transformation (en bas à droite de la figure 2 (2)) qui contient les tâches utiles à la préparation du document : extraction et filtrage de l'information, puis à la création du dossier : édition, composition et formatage. On dispose de tâches élémentaires et de tâches de plus haut niveau réalisant un enchainement de tâches élémentaires. Ces tâches sont génériques et peuvent être combinées selon le besoin. L'utilisateur dispose pour cela d'un langage de composition de tâches conçu à cette fin ; il dispose également d'une liste de tâches prédéfinies et potentiellement utiles pour la communauté d'utilisateurs à laquelle il appartient.

- Le système à base de règles est l'élément central du module de recombinaison (au centre figure 2 (2)). Il utilise la boite à outils en fonction de la demande de l'utilisateur et $\mathrm{du}$ domaine. Les règles modélisent le domaine et les besoins classiques dans celui-ci en terme de production de document ${ }^{8}$ (création d'index, production de pages constituée de fragments ordonnés suivant un certain critère, liens vers des fragments, etc.).

- Une partie est affectée à la gestion des profils : elle gère le profil général des utilisateurs de la communauté (demandes habituelles et préférences générales) et simultanément le profil personnel de l'utilisateur qui peut définir des tâches et des préférences propres ${ }^{9}$. Le système sélectionne en fonction de ces deux profils ${ }^{10}$ les règles de productions destinées à produire le dossier final.

10 L'utilisateur formule d'abord une requête comme dans un moteur de recherche classique, puis choisit une tâche de réédition parmi les tâches possibles correspondant à son profil. Les règles actives sont lancées et amènent à la création du dossier résultat. 


\section{Le problème des textes de lois consolidés}

La production des documents de loi tend de plus en plus à se normaliser pour répondre à des besoins de classement et de consultation (cf. la sixième conférence de la loi via Internet tenue en novembre 2004 à Paris ${ }^{11}$ ) et les systèmes qui donnent un accès en ligne aux textes de lois sont de plus en plus nombreux, ils existent maintenant dans plus de 50 pays.

11 Dans beaucoup de pays, la plupart des lois sont constituées de textes suivis d'une série d'amendements, on les appelle lois consolidées. Bien que celles-ci n'aient pas la même acception légale dans différents pays (valeur instructive contre valeur constitutive), les systèmes offrant une possibilité de manipulation automatique des modifications d'un texte législatif en produisant un document consolidé sont très utiles. EnAct (ArnoldMoore, Clemes, 2000) par exemple, est un système australien d'accès à la législation, qui aide les rédacteurs techniques à produire une rédaction initiale des textes des amendements aux lois (production "initiale ») d'une manière simple et à appliquer des amendements à une loi donnée quand une demande est formulée (production «à la demande »).

En Europe, Eur-Lex offre également des outils pour la navigation dans les documents consolidés manuellement, il supporte vingt langues européennes.

Comme nous le verrons plus loin, une loi consolidée comporte généralement un document principal et des parties ou documents secondaires: les amendements ou les annexes.

Nous présentons ici le module DARES dans le cadre des lois consolidées, l'application qui a servi à le mettre au point et à le tester. Il s'agit ici d'effectuer de la production «à la demande »: produire des documents qui permettent de cheminer au travers des différentes formes valides d'une loi et de visualiser les différentes modifications. On suppose l'existence d'une base de documents XML (une production initiale préexistante) respectant une grammaire connue ou annotés par le module d'annotation.

L'adaptation de notre système générique au domaine des lois a demandé 6 étapes :

- Étape 1 : Écriture de la DTD des documents du domaine et établissement des relations entre les structures. Les éléments XML (fragments) identifiés dans la DTD sont les cibles des opérations de transformation;

- Étape 2 : Conception des règles d'annotation logique qui suivent la DTD et qui étiquettent les fragments logiques;

- Étape 3 : Conception des règles d'annotation sémantique pour identifier l'information comme les relations entre les fragments de documents, les expressions de modifications dans le cas d'amendements. Deux types d'information sont définis : relations (généralisation /spécialisation, etc.) et actions (est remplacé, est inséré, est supprimé, est ajouté) ;

- Étape 4 : Ajout au besoin de méta-données comme la date, l'endroit, l'auteur...

- Étape 5 : Adaptation au domaine des règles d'acquisition et d'évolution de profil prédéfinies;

- Étape 6 : Écriture des règles à utiliser en cascade pour la production du dossier final (tâches de réédition);

La première règle lancée a pour prémisse le but à atteindre et pour conclusion une action qui lance une série de règles en cascade. Cette action est une tâche de haut niveau. Les autres règles détaillent l'enchainement des sous-tâches à effectuer pour la réaliser. Selon 
son métier, l'utilisateur a accès à un ensemble particulier de buts prédéfinis qu'il peut utiliser sans les détailler.

Le système est alors prêt à fonctionner. L'utilisateur n'a plus qu'à décider d'un but (d'une tâche de réédition) et à entrer une requête initiale.

L'utilisateur expert dans le fonctionnement du système peut créer ses propres tâches à partir des tâches élémentaires fournies au départ.

\section{Structure des textes de loi européens}

Connaitre la structure logique des documents manipulés, n'est pas aussi simple qu'il y parait, c'est pourtant un préalable à la formalisation des expressions des amendements dans ces documents. Notre étude est fondée sur des documents de loi extraits des Journaux officiels par l'intermédiaire du système d'Eur-Lex. Les documents, pour l'instant, ne sont malheureusement pas normalisés, ni étiquetés selon une norme spécifique comme ils pourraient l'être si les extensions de TEI ${ }^{12}$ aux textes juridiques ( Finke, 1997)étaient adoptées.

\subsection{Les documents du Journal officiel}

Les textes de Journaux officiels de la Communauté, qui constituent nos données, sont groupés en différentes catégories principales : législation, information et notices, ellesmêmes de différents types: règlements, directives, décisions et recommandations. La nature (REGULATION, etc.) du document est généralement indiquée dans le titre comme nous pouvons le voir dans la figure suivante :

30.9 .2005

Official Journal of the European Union

$\mathbf{L}$

$255 / 1$

I

(Acts whose publication is obligatory)

REGULATION (EC) No 1552/2005 OF THE EUROPEAN PARLIAMENT AND OF THE COUNCIL

of 7 September 2005

on statistics relating to vocational training in enterprises

(Text with EEA relevance)

Figure 3 : Un exemple de régulation

\subsection{Structure des documents et régularité}

La grammaire des documents des Journaux officiels de la Communauté n'est pas directement accessible dans Eur-Lex. Mais nous observons une tendance de normalisation (standardisation) dans la production des documents qui nous a aidés à concevoir une grammaire de document. Par exemple, sur Eur-Lex, nous trouvons les recommandations et les conseils techniques à l'attention de ceux qui établissent les brouillons des lois ${ }^{13}$. La structure proposée ci-dessous résulte de la synthèse des recommandations lues et des 
traits réguliers observés dans les différents textes que nous avons étudiés. Elle ne peut être considérée comme une référence, car elle a été obtenue empiriquement, et est peutêtre imparfaite. Néanmoins, cette grammaire s'est montrée suffisante pour nos besoins courants.

Les actes de la Communauté sont généralement rédigés selon une structure standard détaillée ici :

- le titre : identifie l'acte, il se compose de l'indication du type d'acte, de l'abréviation de la Communauté concernée, du numéro de l'acte et du nom de l'institution ou des institutions qui ont adopté l'acte, de la date de l'adoption, et d'une indication succincte des thèmes;

- le préambule :placé entre le titre et le texte de la loi, il contient des citations et des considérations. Le préambule commence par un nom d'établissement comme TheEuropean Parliament ou The Commission of. Il finit avec une expression présentant le texte de loi qui est habituellement introduit parHave adopted this ;

- les citations : au début du préambule, elles indiquent la base juridique de l'acte, les propositions, les recommandations, les initiatives, les ébauches qui doivent être obtenues, certains avis et d'autres étapes procédurières non-obligatoires. Les citations sont généralement introduites par les expressions consacrées Having regard to ou Acting in accordance with ;

- les considérations :elles forment la partie de l'acte contenant la description des raisons de l'acte ; elles sont placées entre les citations et le texte même. Les considérations sont introduites par Whereas suivi de points numérotés comportant une ou plusieurs phrases complètes. Chaque point commence par un nombre entre les parenthèses comme (1), sauf dans le cas d'une seule considération ;

- le texte de la loi : il est composé d'articles et de points, qui peuvent être groupés en titres, chapitres et sections. La structure des parties est décrite par la figure 4. Un titre ou un symbole non ambigu identifie chaque structure. La figure 4 associe chaque sous-structure à son symbole. Chacune de ces structures finit quand une autre commence. Quand la dernière structure du texte se termine, l'acte lui-même se termine. Le texte commence directement après le préambule et finit avec les mots Done in qui introduisent le lieu et la date de l'édition de l'acte ;

- les annexes : une ou plusieurs annexes commençant par le titre « annexe »sont ajoutées à la fin du document en cas de besoin. Chaque annexe est numérotée : Annexe1, Annexe2, etc. 


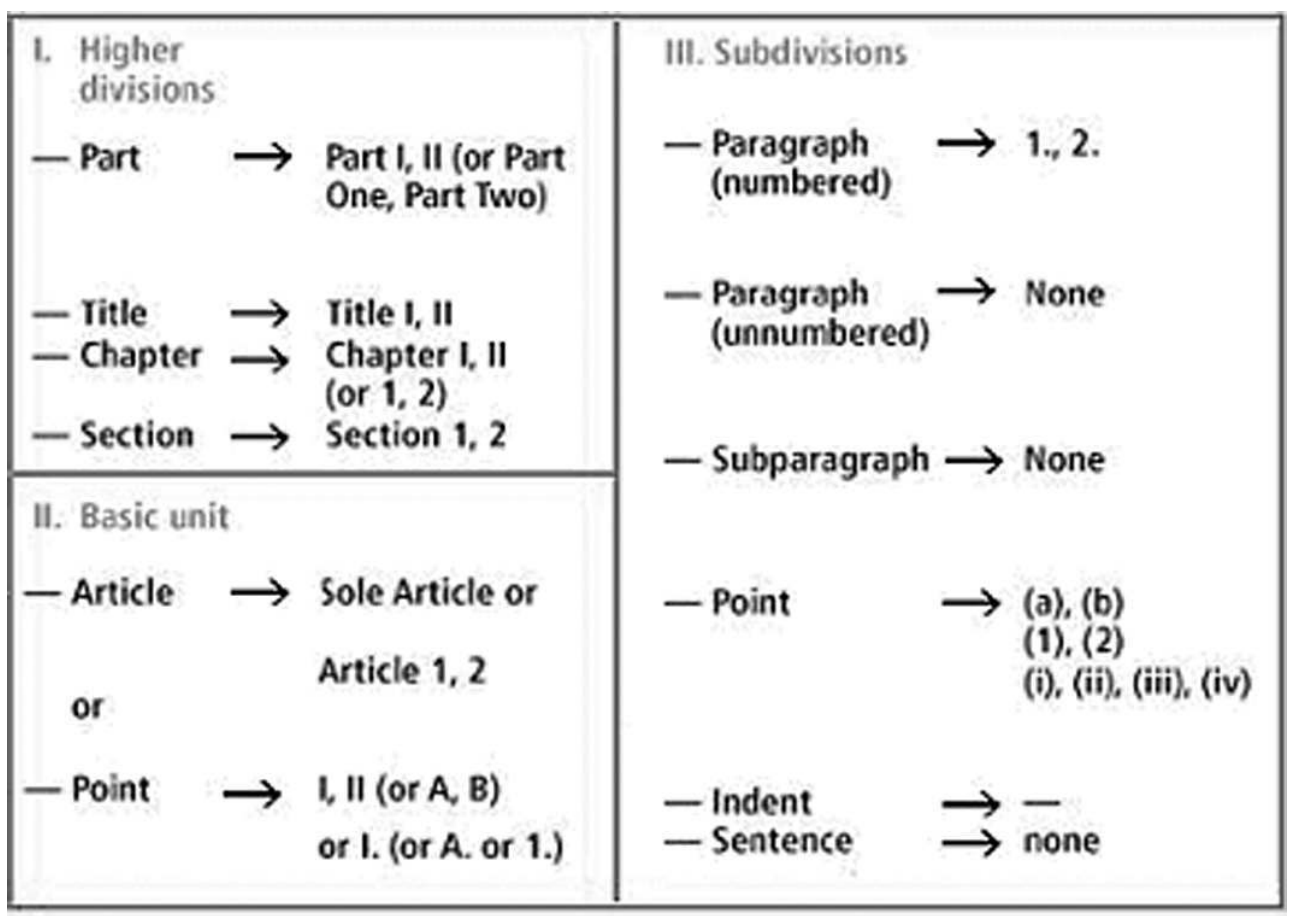

Figure 4 : Structure du texte de la loi

\subsection{Amendements et expressions des révisions}

Les amendements sont normalisés comme tout document. Les techniques législatives de l'office des publications européen donnent quelques recommandations précises pour l'écriture des amendements. Les amendements ne sont pas des documents indépendants, ils ne contiennent pas de nouvelles dispositions substantielles autonomes par rapport à l'acte modifié, ils existent dans le seul but de modifier un acte. Puisque l'effet légal unique du nouvel acte est de modifier l'ancien, il épuise ses effets dès qu'il entre en vigueur.

Généralement les actes de modification ont le même type que l'acte initial. En particulier, on ne recommande pas de modifier un règlement au moyen d'une directive. Cependant, certaines dispositions de la législation primaire laissent le choix du type d'acte aux établissements, en leur accordant le pouvoir d'adopter des mesures ou en mentionnant expressément plusieurs types possibles d'acte.

Le titre de l'acte de modification doit mentionner le numéro de l'acte modifié et, soit indiquer le titre de l'acte, soit indiquer ce qui doit être modifié. Les amendements sont présentés dans le titre par l'expression amending (modification) et le connecteur and, s'il y a plus d'un acte modifié.

Exemple : titre de l'Acte modifié : Council Regulation (EC) No A of... on improving the efficiency of agricultural structures.

Titre de l'amendement : Council Regulation (EC) No B of... amending Regulation (EC) No A on improving the efficiency of agricultural structures.

Là où plusieurs dispositions du même acte doivent être modifiées, tous les amendements sont combinés dans un seul article, comportant une phrase d'introduction et les points suivant l'ordre numérique des articles à modifier. Si plusieurs actes sont modifiés par un 
acte de modification simple, les amendements à chaque acte doivent être présentés ensemble dans un article séparé.

Les modifications des actes sont généralement des textes à insérer dans l'acte à modifier et elles concernent des unités complètes du texte (un article ou une subdivision d'un article) de préférence à différentes phrases ou mots, pour des raisons de clarté et pour faciliter la traduction dans toutes langues officielles.

Les amendements sont faits par l'intermédiaire d'un amendement formel ou textuel. Nous avons répertorié 4 types de modifications formelles :

- suppression : exprimé par les mots is deleted (est supprimé) ;

- remplacement : exprimé par les mots is replaced by (est remplacé par);

- insertion : exprimé par les mots is inserted (est inséré) ;

- ajout : exprimé par les mots is added (est ajouté).

L'expression is amended as follows (est modifié comme suit) présente une liste de modifications formelles.

L'exemple qui suit est celui d'un article d'un acte de modification avec les quatre types de modifications formelles.

Article 1Regulation (EC) No .../... is amended as follows:Article 1 is deleted

Article 23 is amended as follows: a) In paragraph 1, point ( $f$ ) is deleted;

b) Paragraph 2 is replaced by the following: "2. Member States may prescribe that

..."c) The following paragraph $2 a$ is inserted: "2a. In the case of providers of statistical information..."d) The following paragraph 4 is added: "4. The Commission shall ensure publication in the Official Journal...".

\begin{tabular}{|c|c|}
\hline $\begin{array}{c}\text { Type de } \\
\text { Mise à jour }\end{array}$ & 1. Règles de grammaire \\
\hline Deletion & Deletion := Part :('shall' 'be'| 'is') ‘deleted' \\
\hline Adding & Adding := Part ('shall' 'be'| 'is') 'added' \\
\hline Insertion & $\begin{array}{l}\text { Insertion := } \\
\text { ('in' Part (',')? Part ('shall' 'be'| 'is') ‘inserted' ” (('before'| 'after') Part)? )| } \\
\text { (Part ('shall' ‘be'| 'is') 'inserted' ('in' Part) (('before'| 'after') Part)? ) }\end{array}$ \\
\hline Replacement & Replacement := Part ('shall' 'be'| 'is') 'replaced' 'by' Part \\
\hline Part & $\begin{array}{l}\text { Corresponds to the structures identification. A regular expression is generated } \\
\text { by the system according to the DTD given. This expression has been described in } \\
\text { the semantic annotation section. }\end{array}$ \\
\hline
\end{tabular}

Figure 5 : Grammaire d'expression des amendements en $\mathrm{BNF}^{14}$ 


\section{Automatisation de la législation consolidée}

\subsection{Annotation des textes de loi}

La phase d'annotation présuppose qu'une DTD ait été construite et soit entrée dans le système.

Annotation logique : elle est effectuée quand les documents ne sont pas structurés. Il est possible d'utiliser notre outil d'annotation, car les textes d'application sont normalisés. Voici les règles non formelles utilisées pour étiqueter logiquement les documents en anglais ${ }^{15}$.

Étiquetage du titre : si le premier élément des textes produit dans le document contient tous les éléments constitutifs du titre (voir section précédente) et si la marque de début de préambule est trouvée, alors étiqueter le titre.

Étiquetage de préambule : si le titre a été étiqueté et si la marque de début de texte a été trouvée, alors étiqueter le préambule.

Étiquetage du texte législatif : après l'étiquetage du préambule si la marque du début de texte a été trouvée ou la tête d'une sous-structure a été trouvée (article, point, etc.), étiqueter le texte.

Étiquetage de l'annexe : si le marqueur d'annexe a été trouvé et si un autre marqueur d'annexe a été trouvé ou si le document est terminé, étiqueter l'annexe.

Annotation sémantique : selon la grammaire empirique des amendements présentée dans la partie précédente et considérant les possibilités de l'outil d'annotation sémantique générique, nous avons constaté que ce module d'annotation est efficace dans le cadre du droit européen des textes amendements.

L'expert du domaine n'a qu'à alimenter l'outil d'annotation avec une expression régulière portant sur les différentes façons d'exprimer des modifications. Cette expression est la suivante : (shall [ ]+ be $\mid$ is) (deleted $\mid$ replaced [ ]+ by $\mid$ inserted $\mid$ added ).

\subsection{Production du document de synthèse sur demande : premiers résultats}

Dans cette section, nous expliquons les traitements effectués sur les documents structurés pour produire un texte consolidé. Nous allons l'illustrer sur des documents de la base de données Eur-Lex. Pour simplifier, nous supposons que l'utilisateur veuille obtenir un document de synthèse sur spongiform encephalopathies.

Un seul document parle d'encéphalopathie spongiforme. Ce document a pour titre Council Decision of 4 December 2000 concerning certain protection measures with regard to transmissible spongiform encephalopathies and the feeding of animal protein.

Ce document a au moins un acte modificatif nommé "COMMISSION DECISION of 27 March 2002 amending Council Decision 2000/766/EC and Commission Decision 2001/9/EC with regard to transmissible spongiform encephalopathies and the feeding of animal proteins ». Les deux documents sont annotés comme illustré dans la figure 6.

37 Pour générer le document consolidé basé sur ces deux actes, DARES utilise des règles en cascades, écrites au préalable et introduites dans le système dans la phase d'initialisation 
au domaine. La première prémisse est le but par exemple «Make-consolidation-ontopic » faire une consolidation sur un certain thème. Les règles sont :

(1)(make-consolidation-on-topic <user-keywords> <format $>$ )

$\rightarrow$ (extract-fragments-for-consolidation <user-keywords $>$ ) then (merge-extracted) then (format-extracted $<$ format $>$ )

40 La règle (1) est activée par le fait (make-consolidation-on-topic <user-keywords> $<$ format>) quand un utilisateur choisit le but de consolidation proposé par le système, remplit le champ mots-clés de l'interface et indique son format préféré. La règle conduit à l'insertion de trois nouveaux faits dans la base de connaissances. Ces faits activent les règles (2) (4) et (5). Le connecteur «then » est utilisé pour spécifier le fait que les actions se suivent et ne sont pas simultanées.

\section{(2)(extract-fragments-for-consolidation <user-keywords>)}

(initial-docs extract (and (unit « document»)( content ( and (unit « title ») (content $<$ keywords>) ) ) ) ) then (extract-amending-fragments)

\begin{tabular}{|c|c|c|}
\hline \multicolumn{2}{|c|}{ Initial articles } & \multirow{2}{*}{ Same articles with logical and semantic tags } \\
\hline \multicolumn{2}{|c|}{ Article 1} & \\
\hline \multicolumn{2}{|c|}{ 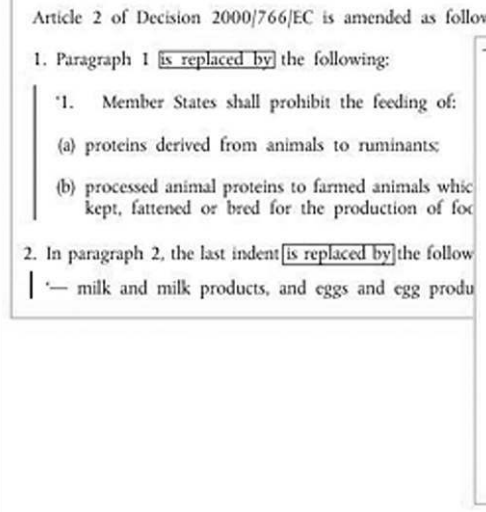 } & 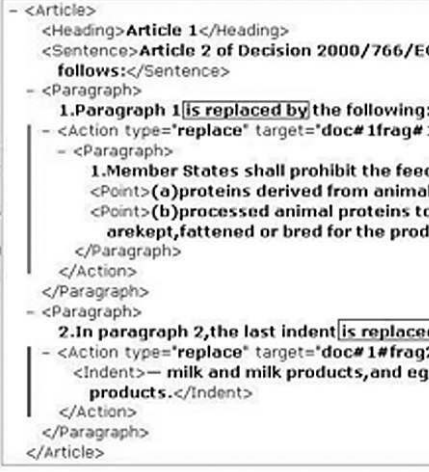 \\
\hline $\begin{array}{l}\text { following: } \\
\begin{array}{|l|}\text { 1. Fishmeal shall be produ } \\
\text { cated only to the produ } \\
\text { which shall be approv } \\
\text { competent authority in : } \\
\text { Council Directive } 90 / 66 \\
\end{array}\end{array}$ & \multicolumn{2}{|c|}{ 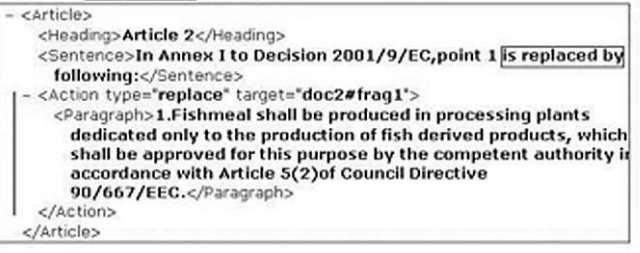 } \\
\hline
\end{tabular}


Amending act articles

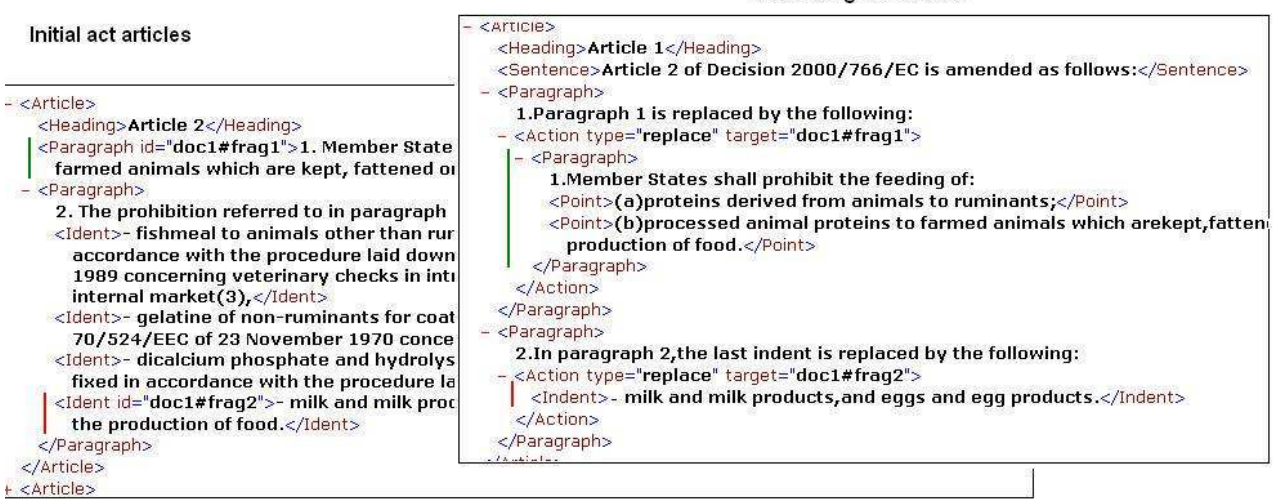

Figure 6 : Étiquetage logique et sémantique des articles du document amendé Décision de la Commission

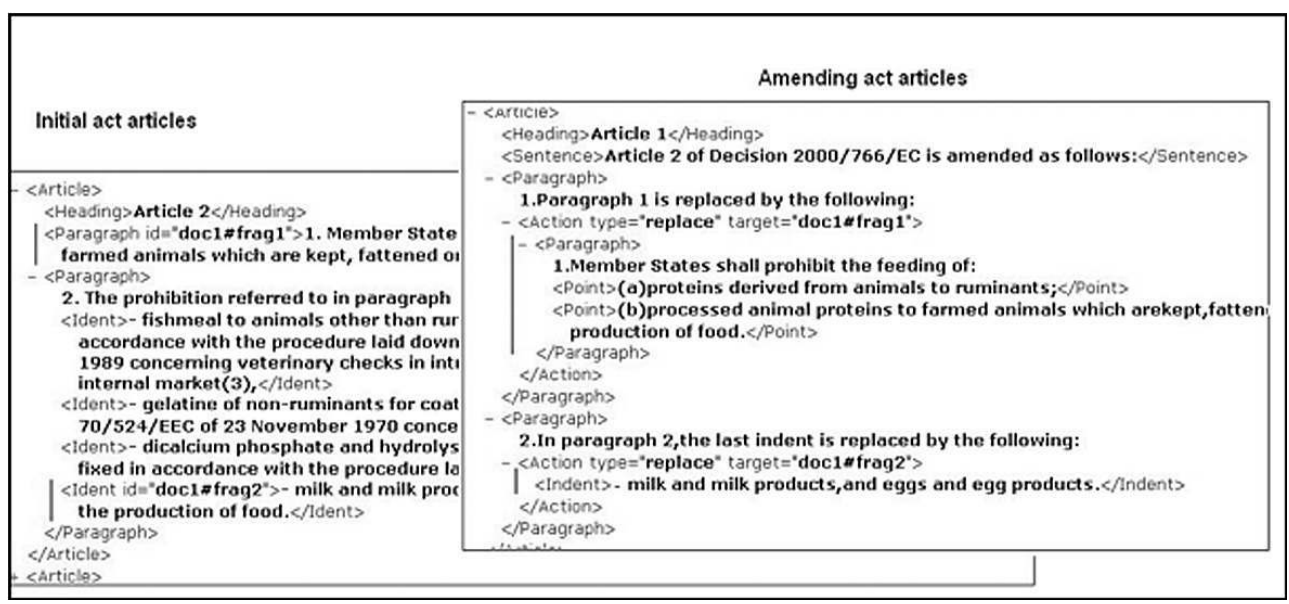

Figure 7 : Acte initial et amendement (XML) effet de fusionner documents et amendements et d'appliquer ces amendements pour produire de nouveaux documents. Elle fait appel à une sous-tâche de remplacement de 
fragments (la tâche "replace») pour réaliser la fusion. Elle ajoute également un index dans le document fusionné obtenu vers les fragments remplacés (en haut de la figure 8).

(5)(format-extracted <format $>$ ) and (Results-docs <docs $>$ )(for-each <docs $>$ format $(<$ format $>,<$ docs $>))$

La dernière règle prend la suite de fragments et les sauvegarde en utilisant un format de sortie (html ou pdf). Une feuille de style par défaut est utilisée à cette fin. Cette feuille de style sera à l'avenir générée pour chaque utilisateur en fonction des préférences de son profil.

La figure 8 illustre un dossier de synthèse résultant de ces règles. En haut de la figure, un index de la révision permet d'accéder à la modification du texte grâce à des liens. Le titre du document modifié est mis en évidence en bleu. Un zoom sur l'article 2 indique les remplacements effectués par la tâche de fusion. Les textes anciens et les nouveaux sont mis en évidence par des couleurs différentes.

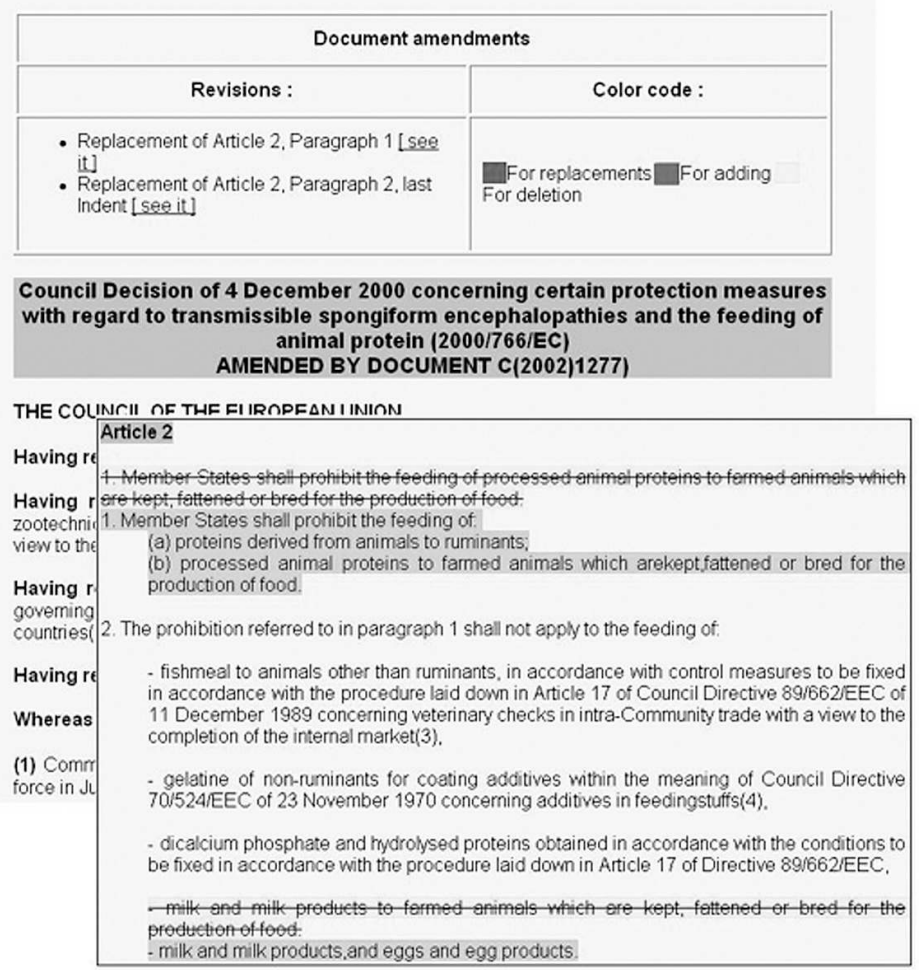




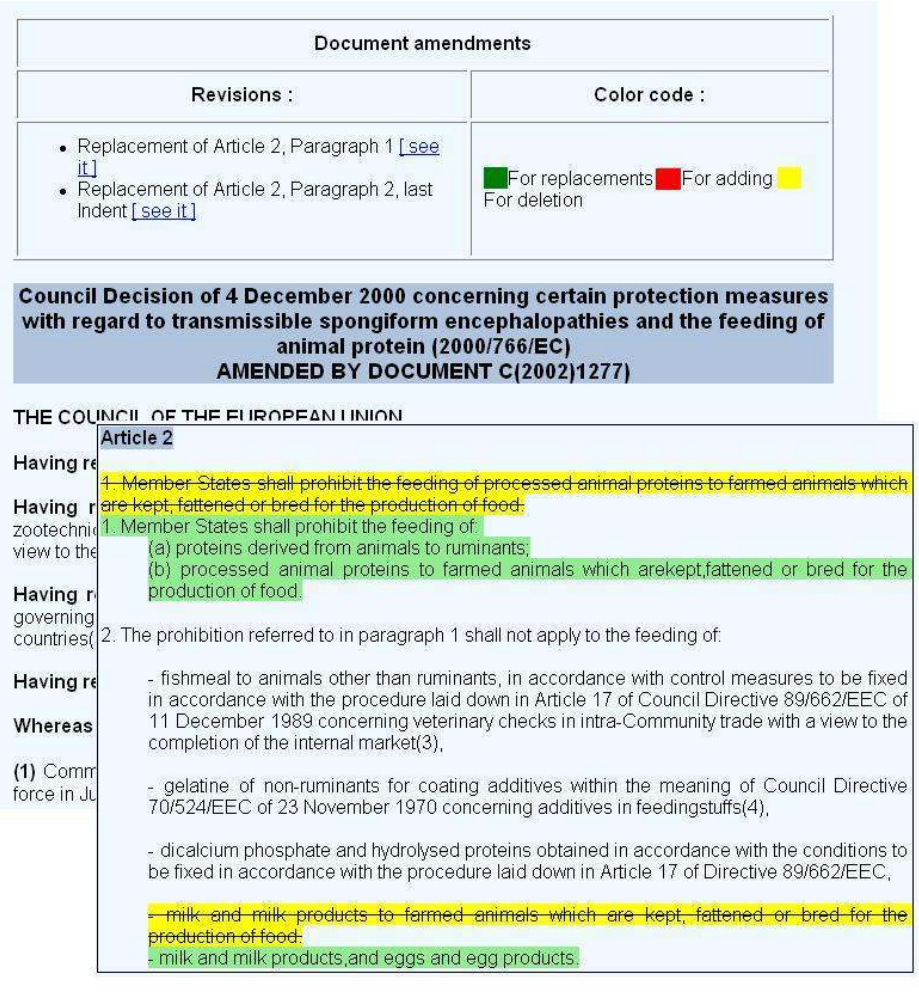

synthèse

Ce résultat montre une possibilité parmi d'autres de génération et de présentation des documents de consolidation. Les règles et les tâches peuvent être enchainées différemment, et beaucoup de présentations sont possibles. Il est possible par exemple de consulter une visualisation du texte valable à une date donnée.

\section{Conclusion et perspectives}

L'élaboration du système PAPLOO nous a conduits à souligner le besoin d'effectuer une annotation des fragments spécifiques à la réédition. Elle nous a amenés à considérer des étiquettes vouées à la réédition dont la sémantique témoignait d'une utilisation possible pour une réorganisation du document (hiérarchie, ordre, relations, etc.) tout en étant génériques, non liées à un domaine. Lors du choix de l'application aux textes juridiques, nous avons été confrontés à un problème nouveau: la manipulation de références à d'autres documents, il nous a donc fallu rajouter les fonctionnalités nécessaires à leur traitement dans le système.

Dans cet article, nous avons proposé une présentation possible des textes consolidés. Ce travail est en cours de validation par des juristes, les premiers résultats sont encourageants. Ce travail doit néanmoins être poursuivi dans plusieurs directions :

- extension à d'autres applications juridiques et tests. Par exemple, il sera intéressant de considérer d'autres types de textes, mais il est vraisemblable que le manque de régularités et d'autres problèmes, liés aux citations, par exemple, poseront des problèmes plus sérieux comme on peut par exemple le voir dans le projet OPENGAVEL qui s'intéresse aux textes de jugements ${ }^{16}$;

- extension à d'autres domaines et tests de transportabilité. Le travail à faire est important ici, il est difficile a priori de qualifier les domaines susceptibles d'être traités facilement, la mise 
en place des outils d'annotations sémantiques demandant toujours un investissement important;

- étude plus approfondie des problèmes posés par la gestion des profils et leur création initiale (Lainé-Cruzel et Guinet, 2000) ;

- création d'une interface ergonomique susceptible de proposer des tâches de réédition à l'utilisateur et de l'aider à en imaginer de nouvelles. Le problème est difficile et un travail important reste à faire à ce sujet. (DARES est une $\mathrm{API}^{17}$ destinée à être incluse dans une chaîne de traitement).

Une nouvelle version de DARES est en cours de développement et commence à répondre à ces questions. DARES est maintenant un plug-in ${ }^{18}$ de la station d'ingénierie des langues LIKES (Rousselot, 2006) et utilise les outils d'annotation de LIKES et son interface. Il possède un début d'interface pour lancer différentes rééditions, il est actuellement testé sur d'autres domaines et notamment sur l'extraction de connaissances à partir de textes de brevets (Ghoula, Khelif, Dieng-Kuntz, 2008).

\section{BIBLIOGRAPHIE}

ARNOLD MOORE T. et CLEMES J. (2000) : Connected to the Law:Tasmanian Legislation Using EnAct, Journal of Information Law and Technology, <http://www2.warwick.ac.uk/fac/soc/law/elj/ jilt/2000_1/arnold>(consulté le 28 Janvier 2008).

BelaÏD A.,ALUSSE A., RANGoni Y., CECotTi H., FARAH F., GAGEAN N., Fiala D., LOGGHE M., VAN DE KERCHOVE K. et MOENS M. F. (2000) : Automatic Version Management of Legislation: The Agora-Lex Project, 11th International Workshop on Database and Expert Systems Applications (DEXA'00), p. 1051-1055. BERTELOOT P. (2004) : Eur-Lex/Celex a new access to the European union laws,Sixth Conference for Law via Internet, Paris,<http://www.frlii.org/spip.php?article79> (consulté le 28 Janvier 2008). FARZINDAR A., LAPALME G. et DESCLÉS J. (2004) : Résumé de textes juridiques par identification de leur structure thématique, Revue TAL nº 45/1, p. 39-64.

FINKE N. D. (1997) : TEI Extensions for Legal Text, TEI 10th Anniversary User Conference. Brown University, Providence, Rhode Island, USA, p. 14-16.

GHOULA N., KHELIF K. et DIENG-KUNTZ R. (2008) : Vers une fouille sémantique des brevets : Application au domaine biomédical, Proceedings de EGC 2008, Revue RNTI,Ed Cepaduès, vol. 11, Sophia-Antipolis, p. 19-30.

LAINÉ-CRUZEL S. et GUINET E. (2000) : Fragmentation et enrichissement de textes scientifiques sous forme électronique, Document Numérique, Éditions Hermès, $4, n^{\circ}$ 1-2, p. 59-84.

RousSelot F. et VIGNE H. (2005) :Document Retro-conversion for Personalized Electronic Reedition , International Workshop on Document Analysis (IWDA 2005), Calcutta.

ROUSSELOT F. (2006) : LIKES un environnement d'ingénierie linguistique et d'ingénierie des connaissances, Formaliser les langues avec l'ordinateur De INTEX à NOOJ, Cahiers de la MSH Ledoux , Presses Universitaire de Franche-Comté, p. 391-411. 


\section{NOTES}

1. En collaboration entre la société informatique Ever Team qui s'occupe de gestion de contenus et d'accès à l'information, et deux laboratoires d'informatique, le LORIA (INRIA Lorraine équipe READ) et le LGECO.

2. OCR : Optical Caracter Recognition, reconnaissance optique de caractères, procédé par lequel un document numérisé sous forme d'image (suite de pixels) peut être converti en suite de caractères.

3. XML: eXtended Markup Language, langage à balises utilisé pour insérer des annotations sémantiques dans des documents Web.

4. HTML : HyperText Markup Language, langage à balises utilisé pour la mise en page et la gestion des liens hypertextes dans les documents Web.

5. Elle consiste principalement à collecter les tâches de recherche liées à la communauté métier et à la fourniture des outils de réédition adaptés.

6. DARES : Documents Annotation and REcombining System.

7. DTD : Document Type Definition. La DTD est une grammaire permettant de vérifier la conformité du document XML, elle définit de manière rigoureuse la structure du document et les types de données qui pourront être intégrées.

8. La séparation entre les opérations génériques de transformation (boite à outils) et l'utilisation de ces opérations par des règles de production de document (système basé sur les règles) garantit l'adaptabilité du système à un domaine particulier.

9. Le profil personnel comporte également le niveau d'expertise dans l'utilisation de DARES qui détermine si l'utilisateur est autorisé à alimenter le système avec de nouvelles règles.

10. Et son niveau d'expertise dans l'utilisation de DARES signale si oui ou non l'utilisateur est autorisé à alimenter le système avec de nouvelles règles.

11. Sixth conference for Law via Internet, November 2004, Paris [en ligne]. Disponible sur : < http://www.frlii.org> (consulté le 30 janvier 2008).

12. TEI : Text Encoding Initiative, La TEI définit une norme de balisage, de notation et d'échange de corpus des documents électroniques.

13. Joint practical guide: Guide of the European Parliament, the Council and the Commission [en ligne]. Disponible sur: <http://europa.eu.int/eur-lex/lex/en/techleg/1.htm> (consulté le 28 janvier 2008).

14. Pour plus d'informations sur les amendements, nous recommandons de consulter le manuel Council's Manual of Precedents, the Commission's Manual on Legislative Drafting and LegisWrite [en ligne]. Disponible sur: <http://europa.eu.int/comm/dgs/translation/workingwithus/freelance/ guide/index_en.htm> (consulté le 28 janvier 2008).

15. Les règles sont également disponibles pour le français.

16. Blog de OPENGRAVEL, Complications in Tagging Legal Citations [en ligne]. Disponible sur : $<$ http://www.opengavel.com/blog1/?p=28> (consulté le 30 janvier 2008).

17. API : Application and Programming Interface. Interface pour la programmation d'applications, il s'agit d'un module sans interface prêt à être intégré dans un plus gros programme.

18. Plug-in : module qui étend les fonctionnalités d'un programme. 


\section{RÉSUMÉS}

L'accès à de grandes bases de données juridiques provoque des besoins particuliers : les requêtes donnent des résultats en trop grand nombre. Le système DARES que nous avons développé propose à l'utilisateur une aide pour réorganiser ces réponses : les documents sont découpés en fragments, les fragments sont ensuite sélectionnés selon certains critères et utilisés pour produire un document de synthèse hyper textuel. Nous décrivons ici une application de ce système relativement générique au traitement de la législation consolidée.

The access to large document bases of legislative texts induces new user needs: answers to user queries are too numerous. The system DARES that was developed by the authors helps the user to re-edit information contained in the returned documents. They are cut into fragments and these fragments are then selected and sorted according to several criteria. The result is a new synthetic hyper-textual document. The example presented here concerns the European Community consolidated laws.

\section{AUTEURS}

FRANÇOIS ROUSSELOT

LGECO INSA Strasbourg

\section{FADY FARAH}

LGECO INSA Strasbourg

\section{AMALIA TODIRASCU}

LILPA Université Marc Bloch Strasbourg 